\title{
Melioidosis Manifesting as Chronic Femoral Osteomyelitis in Patient from Ghana
}

\author{
Diana Ayoola Mabayoje, Dervla T.D. Kenna, David A.B. Dance, Caoimhe NicFhogartaigh
}

\begin{abstract}
A 33-year-old man from Ghana who had diabetes had chronic osteomyelitis of the femoral shaft develop. Tissue samples from surgical debridement grew Burkholderia pseudomallei. He received meropenem, followed by oral trimethoprim/sulfamethoxazole and doxycycline, and fully recovered without complications. Our case report extends the range of countries in Africa as sources of culture-confirmed melioidosis.
\end{abstract}

$\mathrm{M}$ elioidosis is becoming a serious emerging disease worldwide. Burkholderia pseudomallei, the causative agent of melioidosis, is a gram-negative, aerobic bacillus found in wet soil and surface water. Human infection occurs by contact with contaminated soil or water through percutaneous inoculation, inhalation, or ingestion. There is often seasonal variation in incidence in association with heavy rainfall. Human cases are mainly reported in high endemicity areas of Southeast Asia and northern Australia, with sporadic reports from other tropical areas, although past research has predicted that many more areas have the prerequisite climate for B. pseudomallei (1). The purpose of this study was to investigate a case of melioidosis manifesting as chronic femoral osteomyelitis in a patient from Ghana.

\section{The Study}

A 33-year-old man from Ghana who had untreated type 2 diabetes mellitus reported a 2-month history of pain and swelling in the left knee. He had emigrated to the United Kingdom 14 months previously from

Author affiliations: University College London Hospitals National Health Service Foundation Trust, London, UK (D.A. Mabayoje); Public Health England, London (D.T.D. Kenna); University of Oxford, Oxford, UK (D.A.B. Dance); London School of Hygiene and Tropical Medicine, London, (D.A.B. Dance); Barts Health National Health Service Trust, London (C. NicFhogartaigh)
Ghana, where he had lived in a rural northern area (Bolgatanga Province) for 16 months, working as a building project manager. During this period, he traveled to work on a motorbike over unpaved roads. He had occasional night sweats while in Ghana but had no other symptoms and had not sought treatment. He also traveled to urban cities in Nigeria (Lagos and Abuja) as part of his job. He reported visiting Scandinavia, Germany, Brazil, China, and South Africa over the preceding 7 years, although he had always stayed in urban areas and reported no exposure to soil or surface water.

He was febrile (temperature $38.3^{\circ} \mathrm{C}$ ), tachycardic (110 beats/min), and normotensive. His left knee was painful and had a suprapatellar effusion. He had increased levels of inflammatory markers (leukocyte count $11.1 \times 10^{9}$ cells/L, predominantly neutrophils; C-reactive protein level of $221 \mathrm{mg} / \mathrm{L}$; and erythrocyte sedimentation rate of $47 \mathrm{~mm} / \mathrm{h}$ ). Septic arthritis of the left knee was diagnosed. Knee aspirate showed neutrophil polymorphs but a negative Gram stain result and negative cultures. He was empirically given intravenous flucloxacillin, with a plan for a washout. A lytic area was observed on a radiograph of his left femur. Magnetic resonance imaging showed extensive osteomyelitis of the left femoral shaft and metaphysis, including ring enhancement and sinus formation (Figure 1). He underwent incision and debridement of the left femur, during which purulent material was expressed; multiple tissue samples were processed for culture and histologic analysis.

He was empirically given teicoplanin and meropenem. Peripheral blood cultures obtained on the day of hospitalization yielded no growth. Tests results for HIV and syphilis were negative. After 48 hours of culture on standard medium, multiple bone marrow samples yielded an organism identified as $B$. pseudomallei by mass spectrometry. Teicoplanin was stopped, and meropenem started instead. 
The isolate was referred to the National Reference Laboratory for Antimicrobial Resistance and Healthcare-Associated Infections (London, UK), which confirmed B. pseudomallei. MICs determined by gradient diffusion (bioMérieux, https://www.biomerieux. com; Oxoid, https:/ / www.oxoid.com) and interpreted by using European Committee on Antimicrobial Susceptibility Testing breakpoints (https://www. eucast.org). The isolate was susceptible to meropenem, imipenem, doxycycline, ceftazidime, and trimethoprim/sulfamethoxazole. Multilocus sequence typing showed a novel sequence type, ST1914, a single-locus variant of 3 other B. pseudomallei isolates originating in Eritrea, Gabon, and Nigeria (2).

Computed tomography of the chest, abdomen, and pelvis showed no other foci of infection. Because he had labile blood glucose readings and an increased level of hemoglobin A1c, he was given antidiabetic medication. After 14 days of intravenous meropenem, he was switched to oral trimethoprim/sulfamethoxazole (960 mg 2×/d; $160 \mathrm{mg}$ of trimethoprim and $800 \mathrm{mg}$ of sulfamethoxazole) and doxycycline (100 mg 2×/d), completed 2 months of oral antimicrobial drugs, and showed a good clinical response. At the end of treatment, his inflammatory markers had returned to standard levels. Twelve months after he initially sought care, he had full range of movement of his left knee and well-healed surgical scars. He had standard levels of inflammatory markers; a repeat radiograph of his left femur showed changes consistent with his previous debridement and no evidence of ongoing osteomyelitis (Figure 2).

\section{Conclusions}

For our patient, B. pseudomallei infection was probably acquired in Ghana. Melioidosis is underreported in known disease-endemic foci, and modeling has suggested that it is probably endemic to 34 countries that have never reported cases, including 24 in Africa; West Africa was identified as the highest risk area, followed by Central Africa on the basis of environmental suitability $(1,3)$. However, only a handful of sporadic cases have been reported from Africa, probably the result of underdiagnosis caused by resourcelimited laboratories and public health systems (4-9).

Using modeling, Limmathurotsakul et al. estimated an annual incidence of 389 (range 111-1,446) melioidosis cases in Ghana (1). Studies to elucidate the incidence of melioidosis in West Africa, including Ghana, are underway (3). Whole-genome sequencing and phylogenomic analysis of B. pseudomallei isolates have demonstrated that Australia was an early reservoir, with onward transmission to Southeast Asia and
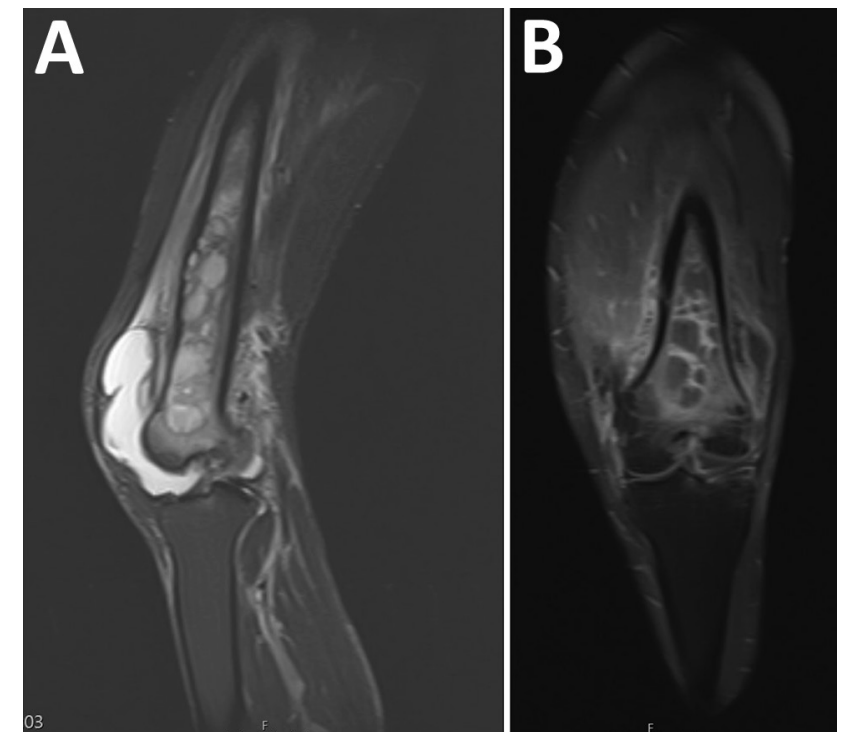

Figure 1. Melioidosis manifesting as chronic femoral osteomyelitis of the left leg in patient from Ghana. A) Magnetic resonance imaging showing extensive femoral osteomyelitis. B) Magnetic resonance imaging showing extensive osteomyelitis.

then to southern Asia. Strains from Africa group into a single clade originating from ancestral clades in Asia and human migration from Indonesia to Madagascar $>2,000$ years ago might have led to dissemination of the organism into Africa with subsequent introduction of B. pseudomallei into the Americas through the transatlantic slave trade (10).

The timing of this patient's manifestations and his history of living in rural areas suggest that he was infected in Ghana. Although he had an extensive travel history, including to other known or potential melioidosis-endemic countries, such as Brazil and Nigeria, he stayed in urban settings and had no rural or soil exposure except in Ghana.

Our patient had untreated diabetes mellitus, a major predisposing factor for melioidosis. Increasing prevalence of diabetes mellitus in Africa means an expected corresponding increase in melioidosis cases. In several countries in Asia, hemoglobinopathies are also associated with increased illness and death caused by melioidosis. The milieu of hyposplenism, defective macrophage and neutrophil chemotaxis, and phagocytosis with iron overload are implicated in the pathogenesis of melioidosis in patients who have thalassemia (11). A recent case series from the Democratic Republic of the Congo described melioidosis in 3 children who had sickle cell anemia, 2 of whom died (12). Hemoglobinopathies are widely prevalent in West and Central Africa and might be an emerging risk factor for melioidosis in settings in Africa. 


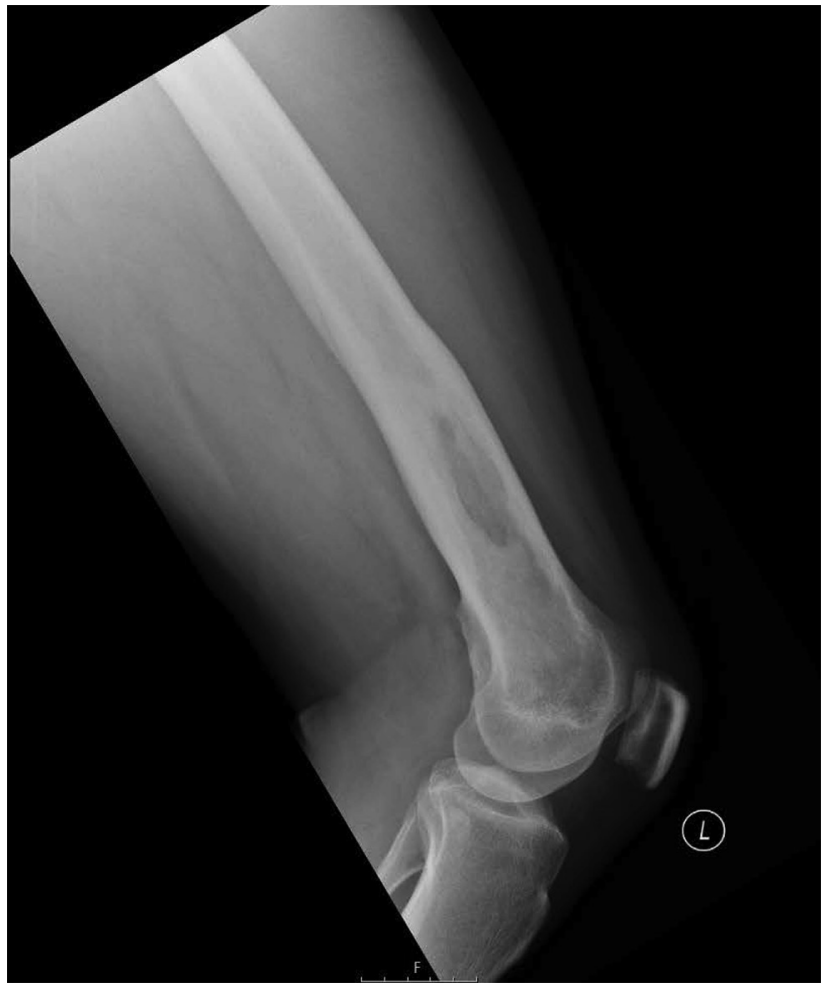

Figure 2. Follow-up radiograph for patient from Ghana with melioidosis manifesting as chronic femoral osteomyelitis of the left leg. Radiograph taken 12 months after initial assessment shows no remaining evidence of infection.

Approximately $7.6 \%-14.4 \%$ of melioidosis cases have musculoskeletal involvement (13-15). A longer duration of infection appears to increase the risk for bone and joint involvement. B. pseudomallei osteomyelitis requires surgical debridement and prolonged treatment with antimicrobial drugs, usually intravenous ceftazidime or a carbapenem, followed by up to 6 months of high-dose, oral trimethoprim/sulfamethoxazole to achieve cure and prevent recurrent infection, which might occur in $\leq 16 \%$ of cases after primary infection (1). In the case we describe, because of a lack of experience with melioidosis osteomyelitis, the patient received only 2 months of dual oral antimicrobial drugs after 2 weeks of intravenous antimicrobial drugs. Despite this suboptimal treatment, he has had no relapse during 12 months of followup. Extensive surgical debridement with good source control might have reduced the risk for recrudescent infection. However, the patient will need to be closely monitored for recurrent infection.

Our case report extends the range of countries in Africa implicated as sources of culture-confirmed melioidosis. We provide additional evidence that melioidosis is underdiagnosed in Africa. This disease should be part of the differential diagnosis of patients with diabetes who have a history of travel in tropical regions and infective symptoms. Strengthening laboratory capacity in Africa will better enable detection of $B$. pseudomallei.

\section{About the Author}

Dr. Mabayoje is a specialist registrar in infectious diseases and microbiology at the University College London Hospital National Health Service Foundation Trust, London, UK. Her primary research interests are the epidemiology of infectious diseases, public engagement, and improved access to healthcare for vulnerable populations.

\section{References}

1. Limmathurotsakul D, Golding N, Dance DA, Messina JP, Pigott DM, Moyes CL, et al. Predicted global distribution of Burkholderia pseudomallei and burden of melioidosis. Nat Microbiol. 2016;1:15008. https:// doi.org/10.1038/ nmicrobiol.2015.8

2. Jolley KA, Bray JE, Maiden MC. Open-access bacterial population genomics: BIGSdb software, the PubMLST.org website and their applications. Wellcome Open Res. 2018;3:124. https:/ / doi.org/10.12688/wellcomeopenres.14826.1

3. Steinmetz I, Wagner GE, Kanyala E, Sawadogo M, Soumeya H, Teferi M, et al. Melioidosis in Africa: time to uncover the true disease load. Trop Med Infect Dis. 2018;3:3. https:// doi.org/10.3390/tropicalmed3020062

4. Morelli F, Smeets L, Hobijn M, Boom H. Melioidosis and renal failure in a Dutch man after a trip to Gambia. Neth J Med. 2015;73:296-8.

5. Salam AP, Khan N, Malnick H, Kenna DT, Dance DA, Klein JL. Melioidosis acquired by traveler to Nigeria. Emerg Infect Dis. 2011;17:1296-8. https:/ / doi.org/10.3201/ eid1707.110502

6. Bremmelgaard A, Bygbjerg I, Høiby N. Microbiological and immunological studies in a case of human melioidosis diagnosed in Denmark. Scand J Infect Dis. 1982;14:271-5. https://doi.org/10.3109/inf.1982.14.issue-4.05

7. Wall RA, Mabey DC, Corrah PT, Peters L. A case of melioidosis in west Africa. J Infect Dis. 1985;152:424-5. https://doi.org/10.1093/infdis/152.2.424a

8. Morosini MI, Quereda C, Gil H, Anda P, Núñez-Murga M, Cantón R, et al. Melioidosis in traveler from Africa to Spain. Emerg Infect Dis. 2013;19:1656-9. https:/ / doi.org/10.3201/ eid1910.121785

9. O'Connor C, Kenna D, Walsh A, Zamarreño DV, Dance D. Imported melioidosis in the United Kingdom: increasing incidence but continued under-reporting. Clin Infect Pract. 2020;7-8:100051. https://doi.org/10.1016/j.clinpr. 2020.100051

10. Chewapreecha C, Holden MT, Vehkala M, Välimäki N, Yang Z, Harris SR, et al. Global and regional dissemination and evolution of Burkholderia pseudomallei. Nat Microbiol. 2017;2:16263. https://doi.org/10.1038/nmicrobiol.2016.263

11. Miraclin AT, Mani SS, Suresh S, Iyyadurai R. Septicemic melioidosis with ruptured splenic abscess in a patient with thalassemia intermedia. J Glob Infect Dis. 2017;9:32-3. https:/ / doi.org/10.4103/0974-777X.194372

12. Shongo M, Yav M, Mukuku O, Kankolongo G, Kumelundu K, Kasongo A, et al. Melioidosis and sickle cell disease: description of a rare association. Theory and Clinical Practice in Pediatrics. 2020;1:57-62. https://doi.org/10.25082/TCPP.2021.01.002 
13. Perumal R, Livingston A, Samuel S, Govindaraju SK. Melioidosis of the musculoskeletal system. Med Princ Pract. 2020;29:121-7. https:// doi.org/10.1159/000503021

14. Morse LP, Smith J, Mehta J, Ward L, Cheng AC, Currie BJ. Osteomyelitis and septic arthritis from infection with Burkholderia pseudomallei: a 20-year prospective melioidosis study from northern Australia. J Orthop. 2013;10:86-91. https://doi.org/10.1016/j.jor.2013.04.001
15. Teparrakkul P, Tsai JJ, Chierakul W, Gerstenmaier JF, Wacharaprechasgu T, Piyaphanee W, et al. Rheumatological manifestations in patients with melioidosis. Southeast Asian J Trop Med Public Health. 2008;39:649-55.

Address for correspondence: Diana A. Mabayoje, Hospital for Tropical Diseases, 2nd Floor, Mortimer Market Centre, Capper St, London WC1E 6JB, UK; email: dmabayoje@nhs.net

\section{December 2020}

\section{Zoonotic Infections}

- Outbreak of Anthrax Associated with Handling and Eating Meat from a Cow Uganda, 2018

- Mycoplasma bovis Infections in Free-Ranging Pronghorn, Wyoming, USA

- Control and Prevention of Anthrax, Texas, 2019

- Animal Rabies Surveillance, China, 2004-2018

- Small Particle Aerosol Exposure of African Green Monkeys to MERS-CoV as a Model for Highly Pathogenic Coronavirus Infection

- Coronavirus Disease Model to Inform Transmission-Reducing Measures and Health System Preparedness, Australia

- Genomic Epidemiology of Severe Acute Respiratory Syndrome Coronavirus 2, Colombia

- SARS-CoV-2 Seroprevalence among Healthcare, First Response, and Public Safety Personnel, Detroit Metropolitan Area, Michigan, USA, May-June 2020

- Flight-Associated Transmission of Severe Acute Respiratory Syndrome Coronavirus 2 Corroborated by Whole-Genome Sequencing

- Risk for Hepatitis E Virus Transmission by Solvent/Detergent-Treated Plasma

- Game Animal Density, Climate, and Tick-Borne Encephalitis in Finland, 2007-2017

- Trends in Population Dynamics of Escherichia coli Sequence Type 131, Calgary, Alberta, Canada 2006-2016 G. Peirano et al. 2907

- Clinical and Multimodal Imaging Findings and Risk Factors for Ocular Involvement in a Presumed Waterborne Toxoplasmosis Outbreak, Brazil

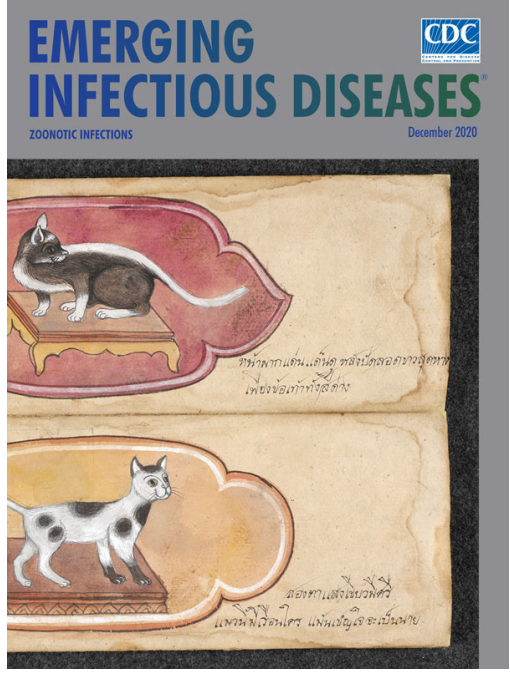

- Outbreak of Haff Disease along the Yangtze River, Anhui Province, China, 2016

- Equine-Like H3 Avian Influenza Viruses in Wild Birds, Chile

- Tuberculosis among Children and Adolescents at HIV Treatment Centers in Sub-Saharan Africa

- Human-Pathogenic Kasokero Virus in Field-Collected Ticks

- Characterization and Source Investigation of Multidrug-Resistant Salmonella Anatum from a Sustained Outbreak, Taiwan

- Outbreaks of H5N6 Highly Pathogenic Avian Influenza (H5N6) Virus Subclade 2.3.4.4h in Swans, Xinjiang, Western China, 2020

- Highly Pathogenic Avian Influenza A(H7N3) Virus in Poultry, United States, 2020
- Differential Tropism of SARS-CoV and SARS-CoV-2 in Bat Cells

- Sensitive Detection of SARS-CoV-2Specific Antibodies in Dried Blood Spot Samples

- Antibody Profiles According to Mild or Severe SARS-CoV-2 Infection, Atlanta, Georgia, USA, 2020

- Experimental Infection of Cattle with SARS-CoV-2

- Susceptibility of Raccoon Dogs for Experimental SARS-CoV-2 Infection

- Zoonotic Pathogens in Ticks from Migratory Birds, Italy

- Coyotes as Reservoirs for Onchocerca Iupi, United States, 2015-2018

- Direct Transmission of Severe Fever with Thrombocytopenia Syndrome Virus from Domestic Cat to Veterinary Personne

- Endovascular Infection with Kingella kingae Complicated by Septic Arthritis in Immunocompromised Adult Patient

- Lymphocytic Choriomeningitis Virus Infections and Seroprevalence, Southern Iraq

- Range Expansion of Bombali Virus in Mops condylurus Bats, Kenya

- Novel Rickettsia Species Infecting Dogs, United States

- Human Monocytic Ehrlichiosis, Mexico City, Mexico

- Hantavirus Cardiopulmonary Syndrome in Canada

- Detection and Characterization of Bat Sarbecovirus Phylogenetically Related to SARS-CoV-2, Japan

\section{EMERGING}

To revisit the December 2020 issue, go to: INFECTIOUS DISEASES https: / / wwwnc.cdc.gov/eid/articles/issue/26/12/table-of-contents 